\title{
Stress Sensors Driving a Feedback Mechanism for the Prediction of Paraspinal Muscle Forces during Upright Stance Posture*
}

\author{
YoungEun $\mathrm{KIM}^{* *}$ and SooTaek KIM*** \\ **Dept. of Mechanical Engineering, Dankook University \\ 126, Jukjeon-dong, Suji-gu, Yongin-si, Gyeonggi-do, 448-701, Korea \\ E-mail:yekim@dankook.ac.kr \\ *** Graduate School, Dankook University \\ 126, Jukjeon-dong, Suji-gu, Yongin-si, Gyeonggi-do, 448-701, Korea
}

\begin{abstract}
For stability analysis of the lumbar spine, a hypothesis is presented that the disc has stress sensors driving a feedback mechanism which can react to the imposed loads by adjusting the contraction of muscles. A three dimensional model of the musculoskeletal system with a detailed lumbar spine finite element model was combined with an optimization technique to calculate muscle forces. The musculoskeletal model consisted of a detailed whole lumbar spine, pelvis, and simplified trunk model. For computational efficiency, the vertebral body and pelvis were modeled as a rigid body and rigid truss elements were used for the rib cage construction. Minimization for deviation of nucleus pressure or Tresca stress in the nucleus was chosen for muscle force calculations. The results indicate that the originally $\mathrm{C}$-shaped lumbar spine was flattened at the upper level, while the more lordotic curvature was generated at the lower level. Muscle forces generated not only in deep muscles, but also in extensor muscles, play an important role in sustaining the lumbar spine to the external load. The resultant forces acting in each vertebra show somewhat different magnitudes and directions compared to the follower load that mimics the deep muscle contraction in in-vitro experiments.
\end{abstract}

Key words: Lumbar Spine, Stability, Muscles, Optimization, Feedback Mechanism

\section{Introduction}

Minor structural differences between the species have a major impact on load-carrying capacity, even though they have similar biological tissues. The curvature of the human lumbar spine is believed to provide a large load-carrying capability. This initial curvature of the spinal column is managed by paraspinal deep muscle activities. The resultant internal compressive force produced with those muscle activities must be tangent to the curve of the spine and is called a follower load ${ }^{(1)}$. Therefore, the follower load is generally applied to incorporate the role of active structures in in-vitro experiments. In the experiments, the follower load is applied bilaterally by cable and dead weight. Many experimental analyses ${ }^{(1)-(4)}$ have shown that the follower load increased spine stability and load-carrying capacity of the spine. The follower load path can also provide a clinically-feasible range of intersegment motion and disc pressure, while sustaining large external loading without buckling ${ }^{(3)}$.

Among the paraspinal deep muscles, it is widely accepted that the lumbar multifidus

*Received 1 Apr., 2008 (No. 08-0266) [DOI: 10.1299/jbse.3.419] 
muscle contributes to stabilization and control of the lumbar spine. Recent work has suggested that the psoas major muscle plays the role of a flexor, as well as a spine stabilizer ${ }^{(5)}$. Moreover, spine stability has predicted that antagonistic muscle co-activation increases stability, which has been confirmed in an electromyographical study ${ }^{(6)}$. Therefore, the follower load is not sufficient to explain the active role in determining the manner in which the applied forces are distributed through the spine.

Some analytical studies were conducted for the effects of the follower load. The studies applied external loads (muscle forces) that minimized the internal shear forces and bending moment, made the resultant internal force compressive, and caused the load path to approximate the tangent to the curve of the lumbar spine ${ }^{(1)}$, or similar to the experimental analysis, the follower load was imposed on the spine finite element model according to the guided direction ${ }^{(2)-(5),(7)}$. The spinal structures are believed to have stress sensors deriving a feedback mechanism, an arrangement that could react to impose loads by modifying muscular action in such a way so as to minimize stress at the joint and therefore the risk of injury ${ }^{(8)}$. Due to this feedback mechanism, stress in the disc will be minimized and equalized. This mechanism will be controlled by muscular activities, which results in lordotic angle changes in the spinal column.

With biomechanical model studies, adequate function of passive and active structures of spinal elements is crucial for partitioning a net reaction role. In this study, we developed a detailed finite element model of the whole lumbar spinal column and identified the physiologic behavior of the disc as a stress senor and the role of the paraspinal muscles for this task.

\section{Material and Methods}

The model consisted of a detailed whole lumbar spine, pelvis, and simplified trunk model. The morphology of the model was based on the Visible Human Project ${ }^{\circledR}{ }^{(9)}$. The lumbar spinal column consisted of five vertebrae and inter-segmental discs. For computational efficiency, the vertebral body was modeled as a rigid body and posterior bony elements were converted into mesh structures, which were composed of hexahedron elements and assigned the same material property values because the amount of cancellous bone is difficult to estimate. The pelvis was also modeled as a rigid body and a rigid truss element was used for the rib cage construction. The model of the annulus fibrosus, an amorphous ground substance which has alternating ring-shaped laminate structures, was composed of annulus ground matrix and reinforced collagen fibers ${ }^{(10)}$. The model was developed by employing the common solid elements and putting the truss elements as layer-upon-layer in diagonal directions. In each layer, the angle of the fiber was about $\pm 34^{\circ}$ with the horizontal axis. The angle of the fiber was taken into account in the modeling so that the area of the fiber was determined to make the total volume of the collagen fibers to be $16 \%$ of the annulus fibrosus ${ }^{(11)}$. The ground substance of the annulus and nucleus was modeled as brick elements with 8 node linear displacement. The simulated model of facet joint articulation was three dimensional interfaces or a gap element model which supported only compression in the direction normal to the surface. For each facet joint, six gap elements were applied in the direction normal to the joint surface. The seven spinal ligaments which connected the vertebrae were modeled as three dimensional, tension-only spar or cable elements ${ }^{(12)}$. The elements were oriented along the direction of the corresponding ligaments. The known material property ${ }^{(13,14)}$ was assigned to each component to complete the model. The constructed model showed that the initial lordosis angle between the superior endplates of L2 and S1 was $43^{\circ}$. The model was validated by comparing the previously reported experimental and FE analysis results ${ }^{(15,16)}$. 


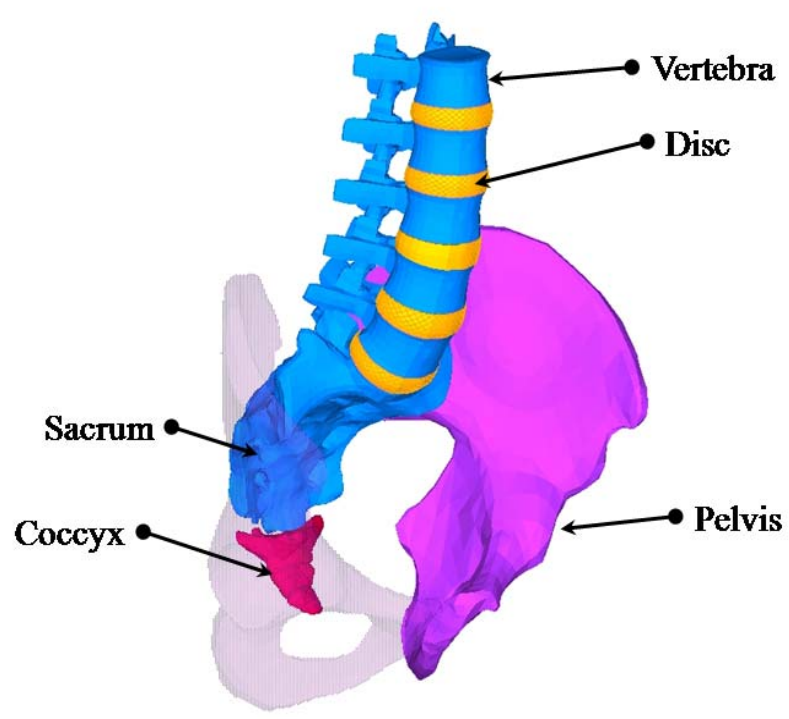

Fig. 1. Developed detailed FE model of the lumbar spine column

Figure 1 indicates a developed detailed lumbar spinal column model with a sacrum and pelvis model. The sacrum and pelvis were modeled as rigid bodies. Therefore, those structures only have a surface shape. The muscle architecture with 46 local muscles was used in this analysis. Three types of back muscles ${ }^{(17)}$ (12 iliocostalis lumborum, 10 longissimus thoracis, and 4 spinalis thoracis) and two types of deep muscles ${ }^{(18)}$ (psoas major and mutifidus) were incorporated to identify the role of the stabilizer. The multifidus has five fascicles that arise from the spinous process and lamina of each lumbar vertebra and descend in a caudolateral direction. Therefore, five pairs of mutifidus were assigned. The psoas major muscle principally supports the trunk on the pelvis. The psoas major muscle is uniquely positioned to prevent buckling of the spine and to control lordosis ${ }^{(5)}$. Five pairs of psoas major were modeled to be connected to each vertebral body. Figure 2 shows a configuration of local musculature in the coronal and sagittal planes.

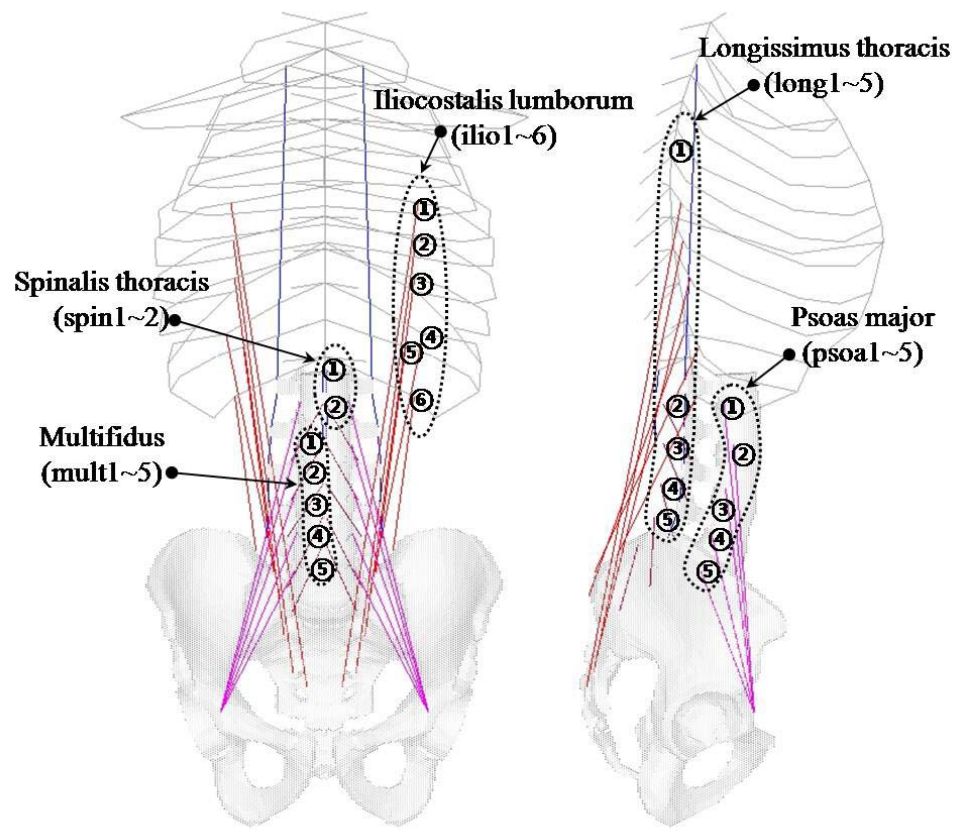

Fig. 2. Representation of local muscle structure in the coronal and sagittal planes. 
The muscle forces were the independent unknowns in the optimization that were calculated by minimizing the cost function. Vertebral motions, disc stress, and nucleus pressure were additional dependent variables in the model that were derived from the detailed FE model and the external load and muscular forces. For this calculation, the finite element method combined with an optimization technique was applied to quantify the role of each muscle and reaction forces in the spinal motion segments for the case of upright stance posture holding 100,200 , or $300 \mathrm{~N}$ weight in hand close to the body. The trunk weight of the model was assumed to be $350 \mathrm{~N}^{(19)}$. In the relaxed upright stance, the body segments are so aligned around the line of gravity that the bending moment and stresses generated by gravity are minimized. We have assumed that upright stance with gravity with weight in hand produces pelvis movement to the posterior direction so that the trunk weight was allocated to the anterior direction.

To investigate the contribution of muscle activation, two different methods, the sequential and direct methods, were conducted. In the sequential method, muscle is assumed to have its own role. For this simulation, two stages of optimization technique were applied because of the classification of the design variable. In the first stage, the applied load given by the trunk weight and weight at hand made the lumbar spinal column flex. From the flexed position, the activation of three types of back muscles made the trunk restore the upright stance posture. In this procedure, back muscle forces can be calculated for maintaining the upright stance posture. With these calculated results, the second stage of minimization was conducted. Minimization of the disc stress (Tresca stress in the nucleus) deviation or nucleus pressure deviation was chosen for the cost function. These two components of the cost function correspond to minimizing the risk of injury at the specific disc level. At this stage, two groups of deep muscles were chosen for design variables. The calculated design variable values adjust the spine curvature, which was derived in the first stage calculation.

In the direct method, it was assumed that muscles contribute not only trunk motion, but lordotic angle changes of the lumbar spine. For this simulation, whole muscle forces have to be determined simultaneously by combing the above two stages of calculation. The multi-cost function was formulated as the weighted sum of the two components as presented in equation (1). The weighting factors, $\alpha$ and $\beta$, are the relative weights of deviation of Tresca stress and pressure of the nucleus, respectively. Proper weighting factors were chosen to reflect the equal contribution of each component.

$$
\operatorname{Min} \mathrm{f}=\alpha \sqrt{\frac{1}{5} \sum_{i=1}^{5}\left(S_{t, i}-S_{t, a v e}\right)^{2}}+\beta \sqrt{\frac{1}{5} \sum_{i=1}^{5}\left(S_{n u, i}-S_{n u, a v e}\right)^{2}}
$$

where,

$\alpha, \beta$; weighting factors

$S_{t, i}$; Tresca stress in the nucleus at the i-th disc

$S_{t, a v e}$; averaged Tresca stress in the nucleus

$S_{n u, i}$; nucleus pressure of the i-th disc

$S_{n u, a v e}$; averaged nucleus pressure

Trunk movement in the anterior-posterior direction was chosen to be a constraint. Tresca stress or nucleus pressure in the each disc level was incrementally evaluated by the non-linear finite element model subject to the external load (trunk weight and weight in hand). These stress or nucleus pressure was supplied into a separate program that calculated muscle forces at each state based on the instantaneous configuration of the lumbar spinal column, including muscle forces generated at the previous iteration. Newly calculated muscle forces were fed into the finite element program to update the stress or pressure level. This iterative approach was continued at each load step until convergence was achieved. 
The finite element program, ABAQUS (version 6.5; Hibbit, Karlsson \& Sorensen, Inc., Pawtucker, RI, USA) was used to carry out non-linear structural analysis on the detailed lumbar spinal column model, while the optimization procedure was performed using the VisualDOC (version 6.0; VR\&D, Inc., Colorado Springs, CO, USA) program.

\section{Results}

By comparing the previously reported experimental and analytical results, our detailed spine model was already validated. The detailed lumbar spinal column model exhibited hypermobility under compressive loading conditions. Application of three types of extensor muscles restored the trunk to its original upright stance posture, which accompanied some curvature change in the lumbar spinal column. Tables 1 and 2 show the calculated extensor and deep muscle forces, respectively.

Table 1. Calculated extensor muscle forces at stage 1 in the sequential method

\begin{tabular}{c|ccc}
\hline \multirow{2}{*}{ Muscles } & \multicolumn{3}{|c}{ Weight in hand } \\
\cline { 2 - 4 } & $100 \mathrm{~N}$ & $200 \mathrm{~N}$ & $300 \mathrm{~N}$ \\
\hline ilio[1] & 36.27 & 56.43 & 76.05 \\
ilio[2] & 33.50 & 52.10 & 70.32 \\
ilio[3] & 30.43 & 47.35 & 63.99 \\
ilio[4] & 24.51 & 38.13 & 51.62 \\
ilio[5] & 23.18 & 36.05 & 48.81 \\
ilio[6] & 17.32 & 26.96 & 36.52 \\
long[1] & 47.45 & 73.75 & 98.83 \\
long[2] & 20.84 & 32.42 & 43.93 \\
long[3] & 15.45 & 24.06 & 32.62 \\
long[4] & 10.18 & 15.82 & 24.48 \\
long[5] & 4.92 & 7.64 & 10.38 \\
spin[1] & 12.98 & 20.19 & 27.36 \\
spin[2] & 24.64 & 38.33 & 51.93 \\
\hline TOTAL & 301.69 & 469.24 & 633.85 \\
\hline
\end{tabular}

ilio: iliocostalis lumborum, long: longissimus thoracis, spin: spinalis thoracis

Table 2. Calculated deep muscle forces at stage 2 in the sequential method

\begin{tabular}{c|ccc|ccc}
\hline \multirow{2}{*}{ Muscles } & \multicolumn{5}{c}{ Cost Function } \\
\cline { 2 - 7 } & \multicolumn{3}{|c}{ Pressure $(\alpha=0, \beta=1)$} & \multicolumn{3}{c}{ Tresca stress $(\alpha=1, \beta=0)$} \\
\cline { 2 - 7 } & \multicolumn{3}{|c}{ Weight in hand } & \multicolumn{3}{c}{ Weight in hand } \\
\cline { 2 - 7 } & $100 \mathrm{~N}$ & $200 \mathrm{~N}$ & $300 \mathrm{~N}$ & $100 \mathrm{~N}$ & $200 \mathrm{~N}$ & $300 \mathrm{~N}$ \\
\hline mult[1] & 2.40 & 4.54 & 9.54 & 6.04 & 0.00 & 2.64 \\
mult[2] & 6.84 & 0.00 & 0.00 & 5.02 & 6.03 & 2.64 \\
mult[3] & 0.53 & 2.55 & 0.00 & 2.79 & 21.61 & 13.42 \\
mult[4] & 4.17 & 3.06 & 2.64 & 5.26 & 26.96 & 54.48 \\
mult[5] & 4.76 & 1.76 & 1.24 & 1.18 & 1.20 & 0.01 \\
psoa[1] & 0.00 & 33.77 & 0.00 & 27.83 & 24.54 & 1.84 \\
psoa[2] & 0.00 & 25.22 & 69.92 & 33.29 & 40.68 & 71.70 \\
psoa[3] & 72.15 & 61.16 & 60.99 & 50.92 & 38.45 & 35.09 \\
psoa[4] & 17.97 & 0.00 & 10.75 & 4.23 & 10.61 & 3.01 \\
psoa[5] & 5.45 & 1.06 & 0.94 & 0.94 & 0.94 & 1.17 \\
\hline Total muscle force & 114.28 & 133.13 & 156.02 & 137.51 & 171.02 & 186.00 \\
\hline
\end{tabular}

mult: multifidus, psoa: psoas major 
Approximately one-third of the total extensor muscle force was required to rearrange the lumbar spinal structure for the case of $100 \mathrm{~N}$ weight in hand and this ratio gradually decreased with additional weight increments.

The generated reaction responses in the lumbar spinal motion segments with activation of the extensor muscles are listed in Table 3. Relatively larger nucleus pressure and stress was generated at the upper level of the lumbar spine, while facet contact appeared only at the lower level. Application of deep muscles (stage 2) created some changes in the motion segment (Tables 4 and 5). Increased compressive forces on the lumbar spinal column with activation of deep muscles created the nucleus pressure and the facet contact force increment. Selection of different cost function produced similar changes in the motion segments.

Table 3. Calculated results in the motion segment after stage 1 in the sequential method

\begin{tabular}{c|ccc|ccc|cccc}
\hline \multirow{2}{*}{$\begin{array}{c}\text { Disc } \\
\text { level }\end{array}$} & \multicolumn{3}{|c|}{ Nucleus pressure (MPa) } & \multicolumn{3}{c|}{ Tresca stress (MPa) } & \multicolumn{3}{c}{ Facet contact force (N) } \\
\cline { 2 - 10 } & $100 \mathrm{~N}$ & $200 \mathrm{~N}$ & $300 \mathrm{~N}$ & $100 \mathrm{~N}$ & $200 \mathrm{~N}$ & $300 \mathrm{~N}$ & $100 \mathrm{~N}$ & $200 \mathrm{~N}$ & $300 \mathrm{~N}$ \\
\hline $\mathrm{L} 1 / 2$ & 0.376 & 0.433 & 0.486 & 0.105 & 0.132 & 0.162 & 0.00 & 0.00 & 0.00 \\
$\mathrm{~L} 2 / 3$ & 0.293 & 0.329 & 0.363 & 0.093 & 0.115 & 0.137 & 0.00 & 0.00 & 0.00 \\
$\mathrm{~L} 3 / 4$ & 0.228 & 0.241 & 0.259 & 0.091 & 0.104 & 0.120 & 0.00 & 0.00 & 0.00 \\
$\mathrm{~L} 4 / 5$ & 0.187 & 0.188 & 0.193 & 0.070 & 0.080 & 0.093 & 81.97 & 61.03 & 40.24 \\
$\mathrm{~L} 5 / \mathrm{S} 1$ & 0.170 & 0.162 & 0.169 & 0.054 & 0.065 & 0.086 & 142.80 & 163.40 & 180.80 \\
\hline AVE & 0.251 & 0.271 & 0.294 & 0.083 & 0.099 & 0.120 & & & \\
STD & 0.076 & 0.099 & 0.117 & 0.018 & 0.024 & 0.028 & & & \\
\hline
\end{tabular}

Table 4. Calculated results in the motion segment after stage 2 in the sequential method $(\alpha=0, \beta=1)$

\begin{tabular}{c|ccc|ccc|ccc}
\hline \multirow{2}{*}{$\begin{array}{c}\text { Disc } \\
\text { level }\end{array}$} & \multicolumn{3}{|c|}{ Nucleus pressure (MPa) } & \multicolumn{3}{c|}{ Tresca stress (MPa) } & \multicolumn{3}{c}{ Facet contact force (N) } \\
\cline { 2 - 10 } & \multicolumn{3}{|c|}{ Weight in hand } & \multicolumn{3}{c}{ Weight in hand } & \multicolumn{3}{c}{ Weight in hand } \\
\cline { 2 - 10 } L1/2 & 0.370 & 0.451 & 0.488 & 0.104 & 0.134 & 0.162 & 0.00 & 0.00 & 0.00 \\
L2/3 & 0.285 & 0.359 & 0.400 & 0.094 & 0.119 & 0.143 & 0.00 & 0.00 & 0.00 \\
L3/4 & 0.253 & 0.297 & 0.317 & 0.097 & 0.116 & 0.130 & 0.79 & 0.00 & 0.00 \\
L4/5 & 0.216 & 0.238 & 0.249 & 0.078 & 0.088 & 0.100 & 128.10 & 93.04 & 88.16 \\
L5/S1 & 0.208 & 0.213 & 0.215 & 0.064 & 0.068 & 0.077 & 161.70 & 183.60 & 209.80 \\
\hline AVE & 0.267 & 0.312 & 0.334 & 0.087 & 0.105 & 0.122 & & & \\
STD & 0.059 & 0.086 & 0.100 & 0.015 & 0.024 & 0.030 & & & \\
\hline
\end{tabular}

Table 5. Calculated results in the motion segment after stage 2 in the sequential method $(\alpha=1, \beta=0)$

\begin{tabular}{c|ccc|ccc|ccc}
\hline \multirow{2}{*}{$\begin{array}{c}\text { Disc } \\
\text { level }\end{array}$} & \multicolumn{2}{|c|}{ Nucleus pressure (MPa) } & \multicolumn{3}{c|}{ Tresca stress (MPa) } & \multicolumn{3}{c}{ Facet contact force (N) } \\
\cline { 2 - 9 } & \multicolumn{3}{|c|}{ Weight in hand } & \multicolumn{3}{c}{ Weight in hand } & \multicolumn{3}{c}{ Weight in hand } \\
\cline { 2 - 10 } L1/2 & 0.384 & 0.441 & 0.484 & 0.106 & 0.133 & 0.162 & 0.00 & 0.00 & 0.00 \\
L2/3 & 0.316 & 0.360 & 0.401 & 0.098 & 0.119 & 0.141 & 0.00 & 0.00 & 0.00 \\
L3/4 & 0.273 & 0.292 & 0.312 & 0.103 & 0.117 & 0.130 & 12.57 & 0.00 & 0.00 \\
L4/5 & 0.230 & 0.240 & 0.255 & 0.082 & 0.095 & 0.112 & 134.70 & 99.65 & 55.72 \\
L5/S1 & 0.222 & 0.209 & 0.222 & 0.068 & 0.072 & 0.096 & 166.20 & 207.20 & 223.80 \\
\hline AVE & 0.285 & 0.308 & 0.335 & 0.091 & 0.107 & 0.128 & & & \\
STD & 0.060 & 0.084 & 0.096 & 0.015 & 0.021 & 0.023 & & & \\
\hline
\end{tabular}


The direct method provides similar results; however, somewhat smaller total muscle forces and reaction forces in the motion segment were generated (Tables 6 and 7). Resultant forces at each vertebra, which are the summation of all muscle forces applied to each vertebra, changed its magnitude and direction, as shown in Figure 3.

Table 6. Calculated muscle forces in the direct method $(\alpha=4, \beta=1)$

\begin{tabular}{|c|c|c|c|}
\hline \multirow{2}{*}{ Muscles } & \multicolumn{3}{|c|}{ Weight in hand } \\
\hline & $100 \mathrm{~N}$ & $200 \mathrm{~N}$ & $300 \mathrm{~N}$ \\
\hline ilio[1] & 33.09 & 43.62 & 69.18 \\
\hline ilio[2] & 24.62 & 40.56 & 57.66 \\
\hline ilio[3] & 17.90 & 28.28 & 43.62 \\
\hline ilio[4] & 13.43 & 22.13 & 30.15 \\
\hline ilio[5] & 14.27 & 16.20 & 33.64 \\
\hline ilio[6] & 9.50 & 19.05 & 25.42 \\
\hline long[1] & 74.04 & 119.85 & 145.25 \\
\hline long[2] & 9.91 & 15.47 & 27.93 \\
\hline long[3] & 10.78 & 23.11 & 26.43 \\
\hline long[4] & 8.56 & 15.66 & 24.34 \\
\hline long[5] & 4.62 & 8.18 & 10.34 \\
\hline $\operatorname{spin}[1]$ & 8.91 & 14.92 & 18.28 \\
\hline $\operatorname{spin}[2]$ & 7.67 & 10.39 & 23.87 \\
\hline multi[1] & 13.64 & 18.57 & 24.85 \\
\hline multi[2] & 13.33 & 23.55 & 25.99 \\
\hline multi[3] & 9.15 & 19.24 & 26.00 \\
\hline multi[4] & 5.57 & 10.37 & 12.94 \\
\hline multi[5] & 1.83 & 3.29 & 3.82 \\
\hline psoas [1] & 20.93 & 29.85 & 32.62 \\
\hline psoas[2] & 28.66 & 43.13 & 50.31 \\
\hline psoas[3] & 16.52 & 28.14 & 31.89 \\
\hline psoas[4] & 7.50 & 11.87 & 14.49 \\
\hline psoas[5] & 3.17 & 4.93 & 6.63 \\
\hline $\begin{array}{c}\text { Total muscle } \\
\text { force }(\mathrm{N})\end{array}$ & 357.72 & 570.37 & 765.66 \\
\hline
\end{tabular}

Table 7. Calculated results in the motion segment in the direct method

\begin{tabular}{c|ccc|ccc|ccc}
\hline \multirow{2}{*}{$\begin{array}{c}\text { Disc } \\
\text { level }\end{array}$} & \multicolumn{3}{|c|}{$\begin{array}{c}\text { Nucleus pressure } \\
(\mathrm{MPa})\end{array}$} & \multicolumn{3}{c|}{ Tresca stress $(\mathrm{MPa})$} & \multicolumn{3}{c}{ Facet contact force (N) } \\
\cline { 2 - 10 } & \multicolumn{2}{|c|}{ Weight in hand } & \multicolumn{3}{c}{ Weight in hand } & \multicolumn{3}{c}{ Weight in hand } \\
\cline { 2 - 10 } & $100 \mathrm{~N}$ & $200 \mathrm{~N}$ & $300 \mathrm{~N}$ & $100 \mathrm{~N}$ & $200 \mathrm{~N}$ & $300 \mathrm{~N}$ & $100 \mathrm{~N}$ & $200 \mathrm{~N}$ & $300 \mathrm{~N}$ \\
\hline L1/2 & 0.328 & 0.371 & 0.428 & 0.087 & 0.102 & 0.128 & 0.00 & 0.00 & 0.00 \\
L2/3 & 0.284 & 0.323 & 0.363 & 0.085 & 0.099 & 0.119 & 0.00 & 0.00 & 0.00 \\
L3/4 & 0.241 & 0.274 & 0.294 & 0.092 & 0.104 & 0.120 & 50.92 & 0.00 & 0.00 \\
L4/5 & 0.210 & 0.230 & 0.240 & 0.075 & 0.084 & 0.096 & 145.00 & 126.10 & 116.40 \\
L5/S1 & 0.210 & 0.215 & 0.211 & 0.065 & 0.066 & 0.073 & 146.10 & 186.90 & 219.20 \\
\hline AVE & 0.255 & 0.282 & 0.307 & 0.081 & 0.091 & 0.107 & & & \\
STD & 0.046 & 0.058 & 0.080 & 0.010 & 0.014 & 0.020 & & & \\
\hline
\end{tabular}




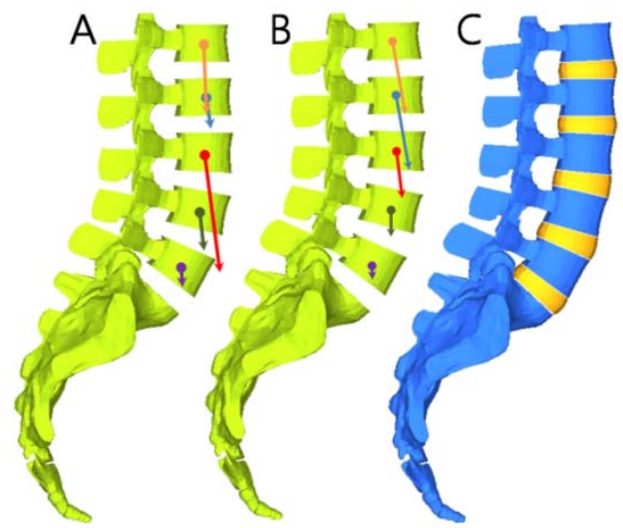

Fig. 3. Resultant muscle forces at each vertebra for the case of $100 \mathrm{~N}$ of weight in hand (arrows indicate the forces direction and relative magnitude). A: sequential method (cost function: pressure deviation), B: direct method $(\alpha=4, \beta=1), C$ : unload
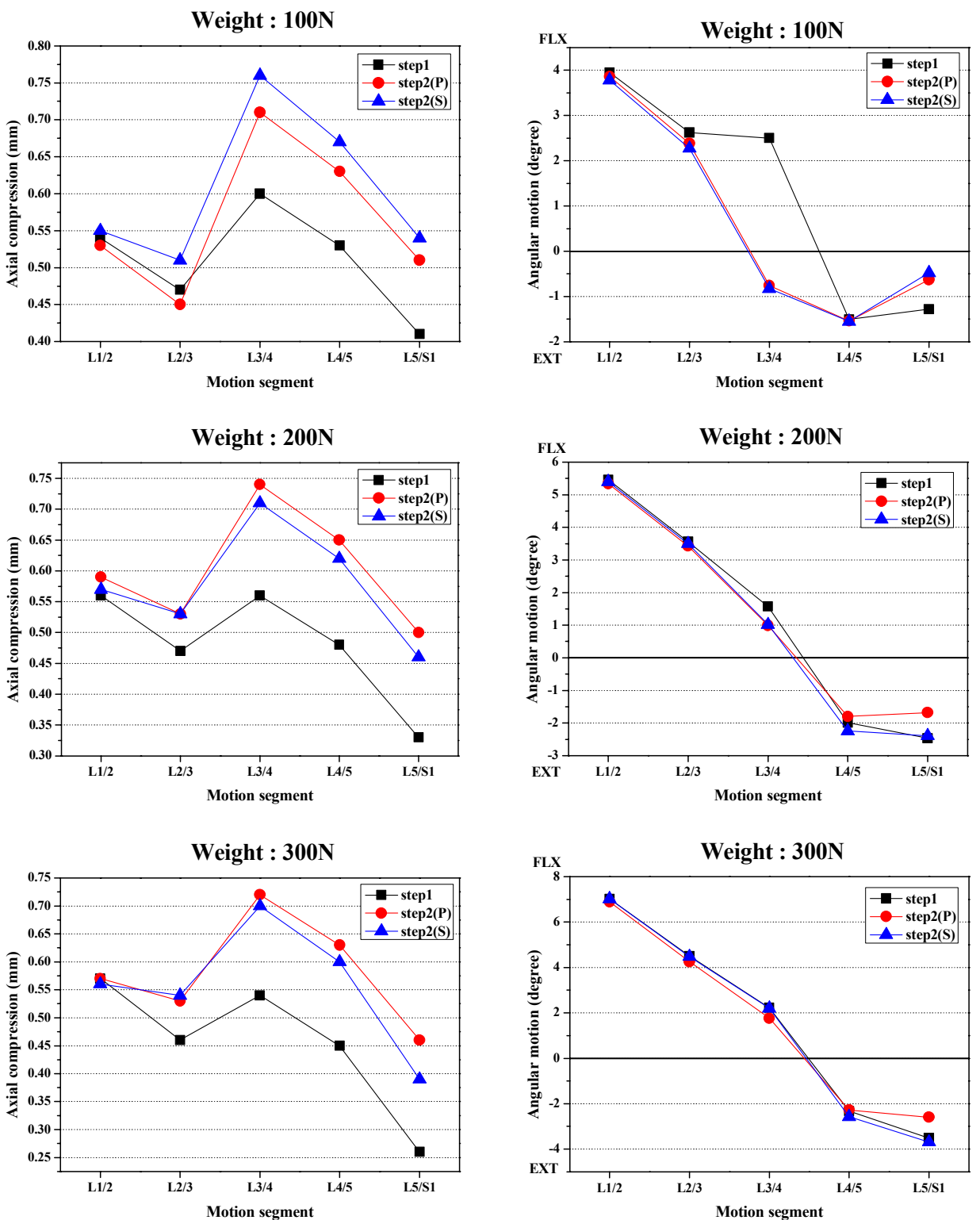

Fig. 4. Motion changes at each motion segment. Steps 1 and 2 mean changes after stages 1 and 2, respectively. (P), (S) indicates the selection of cost function. 
Figure 4 shows the motion changes in each motion segment. These changes were derived from the unloaded initial state so that relatively large motion could be obtained. The upper three motion segments (L1/L4) changed configuration from the extensional position to the flexional position after application of external load and muscle contractions. Opposite to this, the lower two motions segment (L4/S1) increased its original extensional configuration. Additional load (weight in hand) tended to magnify this change. The direct method produced similar patterns of change in axial displacement and angular motion, even though the magnitudes of angular motions were relatively small (Figure 5).
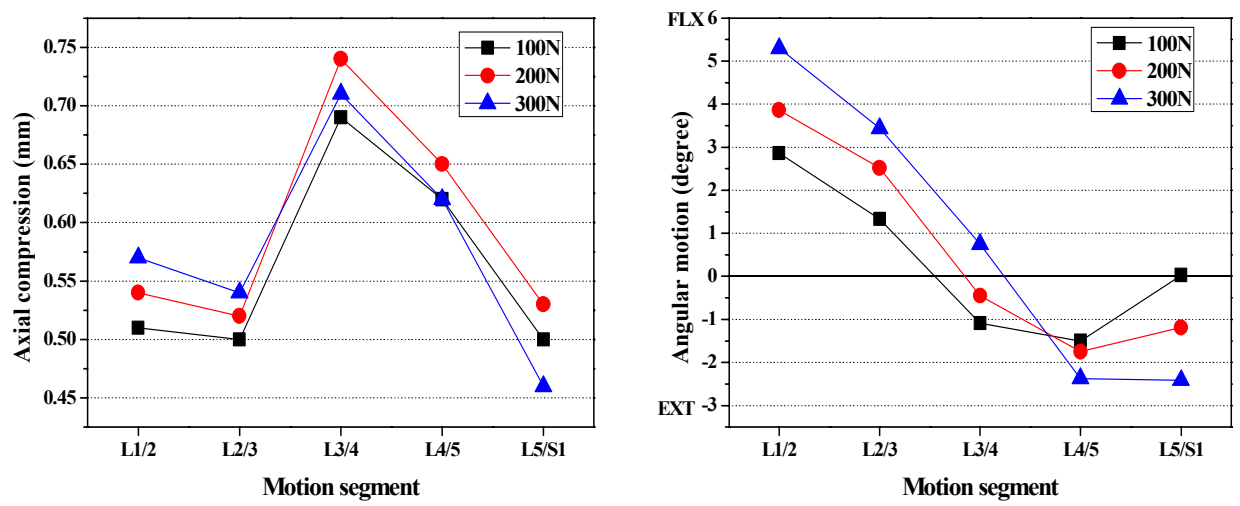

Fig. 5. Motion changes at each motion segment with the direct method.

\section{Discussion}

A compressive load applied to the lumbar spine along the vertical direction caused large deformation at a relatively small load. Several investigators have predicted that the buckling load of columns subjected to the compressive follower load is significantly greater than that of columns under compressive loads along a vertical direction. With this finding, the follower load, by means of cables passed through cable guides attached to the vertebrae, was generally applied to the in-vitro experiment. The goal of this study was to determine the contribution of muscles during stance posture with weight in hand.

Among three types of back muscles for maintaining the upright stance posture, the iliocostalis lumborum played a dominant role, followed by the longissimus thoracis and spinalis thoracis. A total of $165.21 \mathrm{~N}$ was generated in the iliocostalis lumborum, while $98.84 \mathrm{~N}$ was generated in the longissimus thoracis for the case of $100 \mathrm{~N}$ weight in hand. The additional contraction of two types of deep muscles slightly flattened the upper level (L1-L4) of the spinal column. Therefore, the originally C-shaped lumbar spine changed its shape, that is, the upper level lost its lordotic angle, while the more lordotic curvature was generated at the lower level. From the structural point of view, it is reasonable that the straight column could sustain a more compressive load than the curved column without buckling. In contrast to the upper level, the compressive load could be distributed through the facet joint contact and capsular ligament stretching at the lower level (L4-S1) of the lumbar spine. Due the larger facet joint contact forces, smaller nucleus pressure and stress were generated at the lower level. The direct method resulted in a more realistic outcome compared to the sequential method. Shirazi-Adl et al. ${ }^{(20)}$ evaluated muscle forces by minimizing the segmental sagittal moment required for the equilibrium of passive lumbar spine under axial compression. For the given pelvic tilt angle and lumbar lordosis, they derived muscle forces with a simplified beam-rigid body model. Relatively small muscle 
forces were calculated because the axial compressive load was applied mainly at the L1 centre and the rest was distributed equally along the remaining vertebral centre. However, some flattening in the lumbar spine could also reduce the required moments and internal shear force. Similar results were also obtained with the in-vivo experiment ${ }^{(21)}$. Normal volunteers produced lumbar spine flattening and pelvis rotation posteriorly while carry up loads symmetrically in hand in the standing posture.

As the weight in hand increased, the contribution of each muscle in the same muscle group changes its portion of contribution to another muscle. The resultant muscle forces, as illustrated in Figure 3, applied to each motion segment were not parallel to the curvature of the lumbar spine. The force rather acts in an anterior direction instead of a tangential direction. The longissimus thoracis and psoas major muscle forces were the major forces to generate resultant force direction, as seen in Figure 3 (A). In the direct method, addition to those two groups of muscles, the multifidus also contributed to change its resultant force.

Quint et al. ${ }^{(15)}$ was the first to simulate co-activation of muscles with multifidus and psoas major muscles in their experimental analysis. The results of the study underlined the importance of including those muscle groups in in-vitro experiments. An experimental study ${ }^{(5)}$ indicated that the opposite action of the psoas major muscles on the upper and lower lumbar spine regions performed passively and requiring minimal muscular effort, may serve to stabilize the lumbar spine in an upright stance. Our results also suggest that contraction of the multifidus and psoas major muscles mainly attributed these changes and the role for stabilizing the lumbar spine multifidus muscles attached at the upper lumbar spine were important, as shown in Table 6. Most of the in-vitro experiments still have performed with follower load applied by the cable passing through the cable guide attached to the vertebral bodies approximately tangent to the curvature of the spine. This follower load concept applied to the in-vitro experiments acts to mimic optimized deep muscle forces in providing spine stability. Recently, Shirazi-Adl et al. ${ }^{(6)}$ have shown that with the help of a wrapping element, a physiologic load could be applied to the whole lumbar spine. A similar analytical technique was used with thermo-isotropic truss elements ${ }^{(2)}$. But those methods only resembled the in-vitro experimental method, rather than analyzing the muscular structure reaction response to the sensor mechanism of the spine. Our analyses suggest that the combined deep and extensor muscles contribute stability to the lumbar spine column. This result suggests a new avenue to explore the interaction between the muscle and spinal structure, which could not be obtained by previous analysis. However, our analysis could produce some unrealistic outcomes. The calculated nucleus pressure reveals a somewhat smaller magnitude compared to previous reports. Wilke et al. ${ }^{(4)}$ found that vertical load of $260 \mathrm{~N}$ with an additional follower load of $200 \mathrm{~N}$ produced $0.5 \mathrm{MPa}$ nucleus pressure at L2/L3 level in their experimental analysis. The calculated results did not fall within one standard deviation of the in-vitro results. This discrepancy may be attributed to slight variation in disc degeneration, which is not accounted in the model. Viscoelastic behaviour of the disc during in-vivo or in-vitro testing is another factor that was attributed to the difference. The intra-abdominal pressure is believed to increase with the weight supported that should affect the muscle forces. We will include this effect in our future analysis even though its effect would not be dominant.

\section{Conclusions}

For stability analysis of the lumbar spine, a three dimensional model of the musculoskeletal system with a detailed lumbar spine finite element model was developed. The hypothesis presented is that the disc has stress sensors driving a feedback mechanism, which can react to the imposed loads by adjusting the contraction of the muscles. The 
calculated results indicated that deep muscle sincreased the compressive force on the spine, whereas deviation of stress at each disc level was decreased due to those muscle forces. This outcome shows the role of deep muscles as a stabilizer of the spine. Additional research will be continued for a more clear understanding of those muscles in flexion and extension conditions.

\section{Acknowledgments}

This work was supported by the Korea Science and Engineering Foundation (KOSEF) grant funded by the Korea government (MOST; No. R01-2006-000-10257-0).

\section{References}

(1) Patwardhan A. G., Havey R. M., Meade K. P., Lee B., Dunlap B., A follower load increases the load-carrying capacity of the lumbar spine in compression, Spine, Vol. 24 (1999), pp. 1003-1009.

(2) Renner S. M., Natarajan R. N., Patwardhan A. G., Havey R. M., Voronov L. I., Guo B. Y., Andersson G. B., An H. S., Novel model to analyze the effect of a large compressive follower pre-load on range of motions in a lumbar spine, Journal of Biomechanics, Vol. 40, No. 6 (2007), pp. 1326-1332.

(3) Rohlmann A., Neller S., Claes L., Bergmann G., Wilke H. J., Influence of a follower load on intradiscal pressure and intersegmental rotation of the lumbar spine, Spine, Vol. 26 (2001), pp. 557-561.

(4) Wilke H. J., Rohlmann A., Graichen F., Bergmann G., A novel approach to determine trunk muscle forces during flexion and extension; A comparison of data from an inVitro and in-Vivo measurement, Spine, Vol. 28 (2003), pp. 2585-2593.

(5) Penning L., Psoas muscle and lumbar spine stability: a concept uniting existing controversies. Critical review and hypothesis, Eur. Spine Journal, Vol. 9 (2000), pp. 577-585.

(6) Stokes I. A. F., Gardner-Morse M., Lumbar spinal muscle activation synergies predicted by multi-criteria cost function, Journal of Biomechanics, Vol. 34 (2001), pp. 733-740.

(7) Shirazi-Adl A., Parnianpour M., Load bearing and stress analysis of the human spine under a novel wrapping compression load, Clinical Biomechanics, Vol. 15 (2000), pp. 718-725

(8) Gracovetsky S., Function of the spine, Journal of Biomed. Eng., Vol. 8 (1986), pp. 217-223.

(9) Victor M. S., David G. W., Atlas of the visible human male: Reverse engineering of the human body, Jones and Bartlett Publishers Inc., London, (1998).

(10) Lu Y. M., Hutton W. C. and Gharpuray V. M., The effect of fluid loss on the viscoelastic behavior of the lumbar intervertebral disc in compression, Journal of Biomed. Eng., Vol. 120 (1998), pp. 48-54.

(11) Pintar F. A., The biomechanics of spinal elements, Doctoral dissertation, Department of Biomedical Engineering, Marquette University, Milwaukee, (1986).

(12) White III A. A. and Panjabi M. M., Clinical biomechanics of the Spine, Lippincott Williams \& Wilkins, Philadelphia, (1990), pp. 1-83.

(13) Kim Y. E., Goel V.K., Lim T-H., Weinstein J., Effect of disc degeneration at one level on the adjacent level in axial mode, Spine, Vol. 16 (1991), pp. 331-335. 
(14) Kim Y. E., Yun S. S., Effect on the adjacent motion segments according to the artificial disc insertion, Journal of Korean Society of Precision Engineering, Vol. 24 (2007), pp. 122-129.

(15) Yamamoto I., Panjabi M. M., Crisco T., Three dimensional movement of the whole lumbar spine and lumbosacral joint, Spine, Vol. 14 (1989), pp. 1256-1260.

(16) Shirazi-Adl A., Sadouk S., Parnianpour M., Pop D., El-Rich M., Muscle force evaluation and the role of posture in human lumbar spine under compression, Eur. Spine Journal, Vol. 11 (2002), pp. 519-526.

(17) Hansen L., Zee M., Rasmussen J., Andersen T. B., Wong C. and Simonsen E. B., Anatomy and biomechanics of the back muscles in the lumbar spine with reference to biomechanical modeling, Spine, Vol. 31 (2006), pp. 1888-1899.

(18) Santaguida P. L. and McGill M., The psoas major muscle: A three-dimensional geometric study, Journal of Biomechanics, Vol. 28 (1995), pp. 339-345.

(19) Chandler R. F., Clauser C. E., McConvilie J. T., Reynolds H. N. and Young J. W., Investigation of inertial properties of the human body, Final Report, U. S. Dept. of Transportation (1975), pp. 70-74.

(20) Shirazi-Adl A., Parnianpour M., Pelvic tilt and lordosis control: spinal posture response in compression, Trans. Orthop. Res. Soc., Vol. 24 (1999), pp. 1012.

(21) Quint U., Wilke H. J., Shrazi-Adl A., Pamianpour M., Löer F., Claes L. E., Importance of the Intersegmental Trunk Muscles for the Stability of the Lumbar Spine: A Biomechanical Study In-Vitro, Spine, Vol. 23 (1998), pp. 1937-1945. 